\title{
REGULARITY THEOREMS FOR HOLONOMIC MODULES
}

\author{
NAOFUMI HONDA \\ Hokkaido University, Faculty of Science, Department of Mathematics \\ Sapporo, 060 Japan
}

1. Introduction. The notion of holonomic systems entails a natural generalization of ordinary differential equations to higher dimension. A holonomic system is, by definition, a left coherent $\mathcal{E}$ (or $\mathcal{D}$ ) module whose characteristic variety is Lagrangian. It enjoys many good properties (see Kashiwara [7], [8] and Kashiwara-Kawai [10]): for example, all cohomology groups associated with its solution sheaf are constructible. As special functions satisfy systems of ordinary differential equations with regular singularities, a holonomic system with regular singularities introduced by Kashiwara-Oshima [11] and [10] is particularly important. It is well known that the category of holonomic modules with regular singularities is equivalent to that of perverse sheaves through RiemannHilbert correspondence (Kashiwara [9]). Moreover the regularity of holonomic modules is stable under many operations (integration, restriction, etc.). For holonomic modules with irregular singularities, Kashiwara-Kawai obtained the following remarkable theorem.

Theorem [10; Theorem 5.2.1] Let $X$ be a complex manifold and $\mathcal{M}$ a holonomic $\mathcal{E}_{X}$ module. Then there exists a holonomic $\mathcal{E}_{X}$ module $\mathcal{M}_{\text {reg }}$ with regular singularities such that

$$
\mathcal{E}_{X}^{\infty} \otimes_{\mathcal{E}_{X}} \mathcal{M} \simeq \mathcal{E}_{X}^{\infty} \otimes_{\mathcal{E}_{X}} \mathcal{M}_{\text {reg }}
$$

This implies that all holonomic modules are transformed into holonomic modules with regular singularities by use of micro-differential operators of infinite order. The author had shown that this transformation can be achieved by a smaller class of operators (microdifferential operators of Gevrey growth order) corresponding to the irregularity of modules (Honda [7]). Then it is natural to treat a converse problem for holonomic modules (the regularity theorem). The regularity theorem for ordinary differential equations has a long history. Malgrange [16] has shown the regular singularity of the system is equivalent to the convergence of its formal power series solutions. Ramis [17] extend the results

1991 Mathematics Subject Classification: Primary 34A20.

The paper is in final form and no version of it will be published elsewhere. 
to the irregular singular case, that is, the irregularity is characterized by the Gevrey growth order of its formal power series solutions. In the real domain, Komatsu [14] also has a similar result comparing ultra-distribution and hyperfunction solutions. One of the important problems is to extend these results to higher dimensions. The deep study of holonomic systems due to Kashiwara-Kawai [10] and Kashiwara [9] established the regularity theorems for holonomic modules in the regular singular case. The main purpose of this paper is to give several regularity theorem for the irregular singular holonomic modules.

2. Preliminaries. Let $X$ be a complex manifold, $\pi: T^{*} X \rightarrow X$ its cotangent bundle, and $V \subset T^{*} X \backslash X$ a regular involutive or Lagrangian submanifold with the dimension l. We denote by $\mathcal{E}_{X}$ (resp. $\left.\mathcal{E}_{X}(m)\right)$ the subsheaf of $\mathcal{E}_{X}^{\infty}$ consisting of micro-differential operators of finite order (resp. micro-differential operators of order at most $m$ ). For the theory of $\mathcal{E}_{X}$, refer to Sato-Kashiwara-Kawai [18] and Schapira [19]. Now we define the subsheaf $\mathcal{E}_{X}^{(s)}$ of micro-differential operators of Gevrey growth order $(s)$ for any $s \in(1, \infty)$.

Definition 2.1. For an open subset $U$ of $T^{*} X$, a sum $\sum_{i \in \mathbb{Z}} P_{i}(x, \xi) \in \mathcal{E}_{X}^{\infty}(U)$ belongs to $\mathcal{E}_{X}^{(s)}(U)$ if and only if $\left\{P_{i}\right\}_{i \in \mathbb{N}}$ satisfies the following estimate. For any compact set $K$ of $U$, there exists a positive constant $C_{K}$ such that

$$
\sup _{K}\left|P_{i}(x, \xi)\right| \leq \frac{C_{K}^{i}}{i !^{s}}, \quad i>0 .
$$

For convenience, we set $\mathcal{E}_{X}^{(1)}:=\mathcal{E}_{X}^{\infty}$ and $\mathcal{E}_{X}^{(\infty)}:=\mathcal{E}_{X}$.

Next we review briefly the definition of the sheaf of holomorphic microfunctions in Gevrey class. Let $Y$ be a complex submanifold of $X$ and $T_{Y}^{*} X$ its conormal bundle.

Definition 2.2. We define the subsheaf $\mathcal{C}_{Y \mid X}^{\mathbb{R},(s)}$ of the holomorphic microfunctions $\mathcal{C}_{Y \mid X}^{\mathbb{R}}$ in $T_{Y}^{*} X$ as

$$
\mathcal{C}_{Y \mid X}^{\mathbb{R},(s)}:=\mathcal{E}_{X}^{(s)} \mathcal{C}_{Y \mid X}^{\mathbb{R}, f}
$$

where $\mathcal{C}_{Y \mid X}^{\mathbb{R}, f}$ is the sheaf of tempered holomorphic microfunctions (for the definition, refer to Andronikof [1, 2])

Roughly speaking, holomorphic microfunctions in Gevrey class are the holomorphic microfunctions which have Gevrey growth order close to the boundary in the holomorphic representation.

Example 2.3. Let $X=\mathbb{C}$ with a coordinate $(z)$ and $Y=\{z=0\}$. We set for $\epsilon>0$

$$
S_{\epsilon}=\{z \in \mathbb{C} ; 0<|z|<\epsilon,|\arg (z)|<\pi / 2+\epsilon\},
$$

and

$$
\mathcal{O}^{(s)}\left(S_{\epsilon}\right)=\left\{f \in \mathcal{O}\left(S_{\epsilon}\right) \text {; for some } l>0, \sup _{z \in S_{\epsilon}}\left|f(z) \exp \left(-|l z|^{\frac{-1}{s-1}}\right)\right|<\infty\right\} .
$$

The stalk of $\mathcal{C}_{Y \mid X}^{\mathbb{R},(s)}$ at $(0 ;-d z) \in T_{Y}^{*} X$ has the following holomorphic representation:

$$
\left.\mathcal{C}_{Y \mid X}^{\mathbb{R},(s)}\right|_{(0 ;-d z)}=\lim _{\epsilon \rightarrow 0} \frac{\mathcal{O}^{(s)}\left(S_{\epsilon}\right)}{\mathcal{O}_{0}}
$$


Remark that these sheaves are also defined by the functor $T-\mu{ }^{(s)}(\mathcal{O})$, which is a natural extension of tempered microlocalization functor $T-\mu .(\mathcal{O})$ constructed by Andronikof $[1,2]$,

$$
\mathcal{E}_{X}^{(s)}:=\tau^{-1} \tau_{*} T-\mu_{X}^{(s)}\left(\mathcal{O}_{X \times X}\right) \otimes \Omega_{X}[\operatorname{dim} X]
$$

where $\tau: \stackrel{\circ}{T} X \rightarrow P^{*} X$ is the canonical projection, and

$$
\mathcal{C}_{Y \mid X}^{\mathbb{R},(s)}:=T-\mu_{Y}^{(s)}\left(\mathcal{O}_{X}\right)[\operatorname{codim} Y] .
$$

For the definition and properties of the Gevrey microlocalization functor, refer to Honda $[5]$.

To define the irregularity of holonomic modules, we introduce several sheaves. We first define a sheaf of rings $\mathcal{E}_{V}^{(\sigma)} \subset \mathcal{E}_{X}$ for $\sigma$ a rational number $1 \leq \sigma<\infty$. When $\sigma=1$, this sheaf is introduced and studied by Kashiwara-Oshima [11], Kashiwara-Kawai [10] and in general case, by Laurant [15]. We give here the following formulation.

We set the subsheaf $I_{V} \subset \mathcal{E}_{X}(1)$ by

$$
I_{V}:=\left\{P \in \mathcal{E}_{X}(1) ;\left.\delta_{1}(P)\right|_{V} \equiv 0\right\} .
$$

Here we denote the symbol map of degree 1 by $\delta_{1}(\cdot)$. Now we define the sheaf of rings $\mathcal{E}_{V}^{(\sigma)}$ in $\stackrel{\circ}{T} X$

Definition 2.4. For a rational number $\sigma \in[1, \infty)$, we define

$$
\mathcal{E}_{V}^{(\sigma)}:=\sum_{n \geq 0} \mathcal{E}_{X}\left(\frac{(1-\sigma) n}{\sigma}\right) I_{V}^{n} .
$$

In case $\sigma=1$, this sheaf coincides with the sheaf $\mathcal{E}_{V}$ defined in Kashiwara-Oshima [11] and Kashiwara-Kawai [10]. We can find that $\mathcal{E}_{V}^{(\sigma)}$ is stable under the quantized contact transformations. Set $\mathcal{E}_{V}^{(\sigma)}(d)=\mathcal{E}_{X}(d) \mathcal{E}_{V}^{(\sigma)}$ for an integer $d$. We list the properties of the sheaf $\mathcal{E}_{V}^{(\sigma)}$.

(1) $\mathcal{E}_{V}^{(\sigma)}$ is a subring of $\mathcal{E}_{X}$.

(2) $\mathcal{E}_{X}(0) \subset \mathcal{E}_{V}^{(\sigma)}$ and $\mathcal{E}_{V}^{(\sigma)}(d)$ is a left and right $\mathcal{E}_{X}(0)$ module.

(3) $\mathcal{E}_{V}^{(\sigma)}$ is a sheaf of Noetherian ring, and any coherent $\mathcal{E}_{X}$ module is pseudo-coherent over $\mathcal{E}_{V}^{(\sigma)}$.

(4) If $P \in \mathcal{E}_{V}^{(\sigma)}$, then the formal ajoint operator $P^{*}$ belongs to $\mathcal{E}_{V}^{(\sigma)}$.

EXAmPLE 2.5. Let $X=\mathbb{C}^{l}$ with a coordinate system $\left(x_{1}, \ldots, x_{l}\right)$ and

$$
V \subset T^{*} X=\left\{\left(x_{1}, \ldots, x_{l} ; \xi_{1}, \ldots, \xi_{l}\right) ; x_{1}=\xi_{2}=\ldots=\xi_{l}=0, \xi_{1} \neq 0\right\} .
$$

In this case, definition 2.4 is equivalent to the following definition in the above coordinates:

$P \in \mathcal{E}_{X}(m)$ belongs to $\mathcal{E}_{V}^{(\sigma)}(d)$ at $p$ if and only if for the symbol expansion $P(x, \xi)=$ $P_{m}(x, \xi)+P_{m-1}+\ldots$ of $P(x, D)$,

$$
\operatorname{Van}_{(V), p}\left(P_{k}(x, \xi)\right) \geq \sigma(k-d) \quad \text { for } k \leq m \text {. }
$$


Moreover if $\sigma=\frac{q}{p}$ (here $p$ and $q(q \geq p)$ are positive integers and prime to each other)

$$
\begin{aligned}
\mathcal{E}_{V}^{(\sigma)}=\mathcal{E}_{X}(0)\left[\left(x_{1}^{\alpha} \partial_{1}^{\beta_{1}} \ldots \partial_{l}^{\beta_{l}}\right)\right], \quad & 1 \leq k \leq p, \alpha \geq 0 \\
& \beta_{2} \geq 0, \ldots, \beta_{l} \geq 0 \\
& \beta_{1}+\ldots+\beta_{l}=k \\
& \alpha+\beta_{2}+\ldots+\beta_{l}=s_{k} .
\end{aligned}
$$

Here $s_{k}$ is a minimal integer satisfying $s_{k} \geq \sigma k$.

In case that $V \subset T^{*} X=\left\{\left(x_{1}, \ldots, x_{l} ; \xi_{1}, \ldots, \xi_{l}\right) ; \xi_{d}=\ldots=\xi_{n}=0, \xi_{1} \neq 0\right\}$ (the regular involutive case), the same definition as above works. Then we have

$$
\begin{aligned}
\mathcal{E}_{V}^{(\sigma)}=\mathcal{E}_{X}(0)\left[\left(\partial_{1}^{\alpha} \partial_{d}^{\beta_{d}} \ldots \partial_{l}^{\beta_{l}}\right)\right], & 1 \leq k \leq p, \\
& \beta_{d} \geq 0, \ldots, \beta_{l} \geq 0, \\
& \alpha+\beta_{d}+\ldots+\beta_{l}=k, \\
& \beta_{2}+\ldots+\beta_{l}=s_{k} .
\end{aligned}
$$

Here $s_{k}$ is the same as previously.

Let $\mathcal{M}$ be a holonomic $\mathcal{E}_{X}$ module in a neighborhood of $p \in \stackrel{\circ}{T} X$. We first define the weak irregularity of $\mathcal{M}$ at a smooth point of its support. Given $p \notin \operatorname{supp}(\mathcal{M})_{\operatorname{sing}} \cup T_{X}^{*} X$.

Definition 2.6. $\mathcal{M}$ has weak irregularity at most $\sigma$ at $p$ if and only if $\mathcal{M}$ satisfies the following conditions.

There exist an open neighborhood $U$ of $p$, a maximally degenerate involutive submanifold $V$ with its singular locus $\operatorname{supp}(\mathcal{M})$, and an $\mathcal{E}_{V}^{(\sigma)}$ coherent module $\mathcal{M}_{0}$ in $U$ which generates $\mathcal{M}$ over $\mathcal{E}_{X}$ and is finitely generated over $\mathcal{E}_{X}(0)$ at any point of a dense subset in $\operatorname{supp}(\mathcal{M}) \cap U$.

Next we define weak irregularity in the general case.

Definition 2.7. (1) A holonomic $\mathcal{E}_{X}$ module $\mathcal{M}$ has weak irregularity at most $\sigma$ at $p$ if and only if there exist an open neighborhood $U$ of $p$ and a closed analytic subset $Z \supset \operatorname{supp}(\mathcal{M})_{\text {sing }}$ with $\operatorname{codim} Z \geq \operatorname{dim} X+1$ such that, $\mathcal{M}$ has weak irregularity at most $\sigma$ at any point in $U \backslash Z \cap \stackrel{\circ}{T} X$.

(2) A holonomic $\mathcal{D}_{X}$ module $\mathcal{N}$ has weak irregularity at most $\sigma$ if and only if $\mathcal{E}_{X} \otimes_{\mathcal{D}_{X}} \mathcal{N}$ has irregularity at most $\sigma$ at any point in $\stackrel{\circ}{T} X$.

We give several lemmas and examples for the weak irregularity.

LEMma 2.8. Let $0 \rightarrow \mathcal{M}_{1} \rightarrow \mathcal{M}_{2} \rightarrow \mathcal{M}_{3} \rightarrow 0$ be an exact sequence of holonomic $\mathcal{E}_{X}$ modules. Then $\mathcal{M}_{2}$ has weak irregularity at most $\sigma$ if and only if $\mathcal{M}_{1}$ and $\mathcal{M}_{3}$ have weak irregularity at most $\sigma$.

ExAmple 2.9. Let $X=\mathbb{C}, V=T_{\{0\}}^{*} X\left(\subset T^{*} X\right)=\{(x ; \xi) ; x=0, \xi \neq 0\}, q=0$ and $p=(0 ; d x)$. Let $P(x, D)=x^{d} D^{m}+a_{m-1}(x) D^{m-1}+\ldots+a_{0}(x)$ be a differential operator of order $m$. We define the rational number $\operatorname{Irr}_{V, q}(P)$ by

$$
\operatorname{Irr}_{V, q}(P)=\max \left\{1, \frac{d-\operatorname{Van}_{\{0\}, q}\left(a_{k}(x)\right)}{m-k}\right\} .
$$


Then the holonomic $\mathcal{E}_{X}$ module $\frac{\mathcal{E}_{X}}{\mathcal{E}_{X} P}$ has weak irregularity at $\operatorname{most} \operatorname{Irr}_{V, p}(P)$. Conversely we obtain the following

LEMMA 2.10. Let $\mathcal{M}$ be a holonomic $\mathcal{E}_{X}$ module which has weak irregularity at most $\sigma=\frac{q}{p}$ at $p$. Then for any $u \in \mathcal{M}$, there exists a differential operator $P$ such that $P u=0$ and $\operatorname{Irr}_{V, p}(P) \leq \sigma$.

Remark that in spite of $\mathcal{M}$ being an $\mathcal{E}_{X}$ module, we can find such an operator in the category of differential operators.

We investigate the functoriality of the irregularity by the inverse image. Let $f: Y \hookrightarrow$ $X$ be an inclusion of two complex manifolds. We associate the morphisms $\varpi$ and $\rho$ as usual:

$$
T^{*} Y \leftarrow T^{*} X \times_{Y} Y \rightarrow T^{*} X .
$$

LEMMA 2.11. Let $\mathcal{M}$ be a holonomic $\mathcal{E}_{X}$ module which has weak irregularity at most $\sigma$. If $\mathcal{M}$ is non-characteristic along $Y$, then $\mathcal{M}_{Y}=\rho_{*}\left(\mathcal{E}_{Y \rightarrow X} \otimes_{\varphi^{-1} \mathcal{E}_{X}} \varphi^{-1} \mathcal{M}\right)$ has weak irregularity at most $\sigma$.

3. Statement of main theorem. We give our main theorems. Let $X$ be a complex manifold with its contangent bundle $T^{*} X$.

Main TheOrem 1. Let $U \subset T^{*} X$ be a $\mathbb{C}^{\times}$conic open set, $\mathcal{M}$ a holonomic $\mathcal{E}_{X}$ module in $U$ and $\sigma \geq 1$ a rational number. The following conditions (1), (2) and (3) are equivalent.

(1) There exists a holonomic $\mathcal{E}_{X}$ module $\mathcal{M}_{\text {reg }}$ with regular singularities satisfying

$$
\mathcal{E}_{X}^{(s)} \otimes_{\mathcal{E}_{X}} \mathcal{M} \simeq \mathcal{E}_{X}^{(s)} \otimes_{\mathcal{E}_{X}} \mathcal{M}_{\text {reg }}
$$

in $U$ for all $s \in\left[1, \frac{\sigma}{\sigma-1}\right]$.

(2) For any submanifold $Y \subset X$ and any $s \in\left[1, \frac{\sigma}{\sigma-1}\right]$, we have

$$
\left.\left.\mathbb{R} \mathcal{H o m}_{\mathcal{E}_{X}}\left(\mathcal{M}, \mathcal{C}_{Y \mid X}^{\mathbb{R},(s)}\right)\right|_{U} \simeq \mathbb{R} \mathcal{H o m}_{\mathcal{E}_{X}}\left(\mathcal{M}, \mathcal{C}_{Y \mid X}^{\mathbb{R}}\right)\right|_{U}
$$

(3) $\mathcal{M}$ has weak irregularity at most $\sigma$ in $U$.

We give a short explanation of the meaning of the main theorem. (3) to (1) means, as mentioned in the introduction, any holonomic modules (with irregular singularities) can be transformed into regular singular holonomic modules by micro-differential operators of Gevrey growth order corresponding to irregularities of modules. The statement (1) in the theorem is a quite powerful tool to study the growth order of solutions, and for its application we give corollaries 1, 2 below. (2) to (3) implies the irregularity of a holonomic module is controlled by the growth order of its solutions, as shown by many mathematicians in the case of ordinary differential equations.

We give some corollaries. Let $M$ be a real analytic manifold with complexification $X$.

Corollary 1. Let $\mathcal{M}$ be a holonomic $\mathcal{E}_{X}$ module at $p \in T^{*} X$ with weak irregularity at most $\sigma$. Then we have the isomorphisms for all $s \in\left[1, \frac{\sigma}{\sigma-1}\right]$,

$$
\mathbb{R} \mathcal{H o m}_{\mathcal{E}_{X}}\left(\mathcal{M}, \mathcal{C}_{M}^{(s)}\right) \simeq \mathbb{R} \mathcal{H} m_{\mathcal{E}_{X}}\left(\mathcal{M}, \mathcal{C}_{M}\right)
$$


where $\mathcal{C}_{M}$ and (resp. $\mathcal{C}_{M}^{(s)}$ ) is the sheaf of microfunctions (resp. microfunctions of Gevrey class $(s))$.

In the case that $\mathcal{M}$ is regular singular and the solution sheaf is tempered microfunctions, this result was already obtained by Andronikof [3]. We also remark that applying the functor $\tau_{*}: \stackrel{\circ}{T} X \rightarrow P^{*} X$ to the result (2) of the main theorem, we can recover the results of Laurent [15].

Corollary 2 [15]. Let $\mathcal{M}$ be a holonomic $\mathcal{E}_{X}$ module at $p \in T^{*} X$ with weak irregularity at most $\sigma$. Then we have the isomorphisms for all $s \in\left[1, \frac{\sigma}{\sigma-1}\right]$ and for any submanifold $Y \subset X$,

$$
\mathbb{R H}_{\mathcal{H}} \operatorname{com}_{\mathcal{E}_{X}}\left(\mathcal{M}, \mathcal{C}_{Y \mid X}^{(s)}\right) \simeq \mathbb{R} \mathcal{H} \operatorname{om}_{\mathcal{E}_{X}}\left(\mathcal{M}, \mathcal{C}_{Y \mid X}^{\infty}\right)
$$

We have also the distribution version of the above theorem. Let $X$ be the complexification of a real analytic manifold $M$ and $\mathcal{N}$ a holonomic $\mathcal{D}$ module at $q \in M$. We denote by $\mathcal{D} b_{M}^{(s)}$ (resp. $\mathcal{B}_{M}$ ) the sheaf of ultra-distributions of Gevrey class ( $s$ ) (resp. Sato's hyperfunctions) in $M$.

\section{Main Theorem 2. Assume}

$$
\left(\operatorname{char}(\mathcal{N})_{\text {reg }} \cap T_{M}^{*} X\right)^{\mathbb{C}}=\operatorname{char}(\mathcal{N})_{\text {reg }} .
$$

(This means the smooth part of the characteristic variety is the complexification of a real analytic Lagrangian variety in $T_{M}^{*} X$.) Then the following conditions (1), (2) and (3) are equivalent.

(1) There exists a holonomic $\mathcal{D}_{X}$ module $\mathcal{N}_{\text {reg }}$ with regular singularities satisfying

$$
\mathcal{D}_{X}^{(s)} \otimes_{\mathcal{D}_{X}} \mathcal{N} \simeq \mathcal{D}_{X}^{(s)} \otimes_{\mathcal{D}_{X}} \mathcal{N}_{\text {reg }}
$$

in a neighborhood of $q$.

(2) For any $s \in\left[1, \frac{\sigma}{\sigma-1}\right]$, we have

$$
\mathbb{R} \mathcal{H o m}_{\mathcal{D}_{X}}\left(\mathcal{N}, \mathcal{D} b_{M}^{(s)}\right) \simeq \mathbb{R} \mathcal{H o m}_{\mathcal{D}_{X}}\left(\mathcal{N}, \mathcal{B}_{M}\right)
$$

in a neighborhood of $q$.

(3) $\mathcal{N}$ has weak irregularity at most $\sigma$ in a neighborhood of $q$.

The main theorem 2 is the extension to higher dimensions of the following result of H. Komatsu [14].

Let $P$ be an ordinary differential operator. Then $\operatorname{Irr}(P)<\sigma$ if and only if for any $s \in\left[1, \frac{\sigma}{\sigma-1}\right]$, the hyperfunction solutions of $P$ and the ultra-distribution solutions in Gevrey class $(s)$ coincide.

\section{References}

[1] E. Andronikof, Microlocalisation tempérée des distributions et des fonctions holomorphes I, C. R. Acad. Sci. Paris 303 (1986), 347-350.

[2] - Microlocalisation tempérée des distributions et des fonctions holomorphes II, ibid. 304 (1987), 511-514. 
[3] E. Andronikof, On the $\mathcal{C}^{\infty}$-singularities of regular holonomic distributions, Ann. Inst. Fourier (Grenoble) 42 (1992), 695-704.

[4] N. Honda, On the reconstruction theorem of holonomic modules in Gevrey classes, Publ. R.I.M.S. 27 (1991), 923-943.

[5] -, Microlocalization in Gevrey classes, in preparation.

[6] - Regularity theorems for holonomic modules, in preparation.

[7] M. Kashiwara, On the maximally overdetermined systems of linear differential equations, I, Publ. R.I.M.S. 10 (1975), 563-579.

[8] —, On the holonomic systems of linear differential equations, II, Invent. Math. 49 (1978), 121-135.

[9] —, The Riemann-Hilbert problem for holonomic systems, Publ. R.I.M.S. 20 (1984), 319-365.

[10] M. Kashiwara and T. Kawai, On the holonomic systems of microdifferential equations, III, ibid. 17 (1981), 813-979.

[11] M. Kashiwara and T. Oshima, Systems of differential equations with regular singularities and their boundary value problems, Ann. of Math. 106 (1977), 145-200.

[12] M. Kashiwara and P. Schapira, Microlocal study of sheaves, Astérisque 128 (1985).

[13] —, - Sheaves on Manifolds, Grundlehren Math. Wiss. 292, Springer, 1990.

[14] H. Komatsu, On the regularity of hyperfunction solutions of linear ordinary differential equations with real analytic coefficients, J. Fac. Sci. Univ. Tokyo Sec. IA 20 (1973), 107-119.

[15] Y. Laurent, Théorie de la deuxième microlocalisation dans le domaine complexe, Progr. Math. 53, Birkhäuser, 1985.

[16] B. Malgrange, Sur les points singuliers des équations différentielles, Enseign. Math. 20 (1974), 147-176.

[17] J.-P. Ramis, Devissage Gevrey, Astérisque 59-60 (1978), 173-204.

[18] M. Sato, T. Kawai and M. Kashiwara, Hyperfunctions and pseudodifferential equations, in: Lecture Notes in Math. 287, Springer, 1973, 265-529.

[19] P. Schapira, Microdifferential Systems in the Complex Domain, Grundlehren Math. Wiss. 269, Springer, 1985. 